\title{
Original
}

[Journal of Environmental Chemistry Vol.14, No.3, pp.625-632, 2004]

\section{Extraction Condition and Efficiency of Some Hazardous Organic Chemicals from Aqueous Solutions Using a Thermoresponsive Polymer System}

\author{
Kazuho INABA, Masami KANAO-KOSHIKAWA, Taeko DOI and Takashi YAMAMOTO \\ National Institute for Environmental Studies \\ (16-2 Onogawa, Tsukuba, Ibaraki 305-8506 Japan)
}

[Received February 6, 2004]

\begin{abstract}
Summary
The extraction behavior of $\beta$-estradiol, bisphenol $A$ and triclosan observed in a thermoresponsive poly(vinylmethyl) ether precipitate system were investigated. The three toxicants were extracted from aqueous solutions into a gum-like precipitate, which formed by aggregation when the solution was heated to a temperature higher than the lower critical solution temperature of the polymer (ca. $305 \mathrm{~K}$ ). An anionic surfactant, linear dodecylbenzenesulfonate was found to decrease the efficiency in formation of the precipitate and in extraction of the target chemicals. However, no such effect was found by addition of a kind of nonionic surfactant polyethyleneglycol tert-octylphenyl ether or humic acid, which are typical coexisting organic materials in environmental waters. More than $90 \%$ of the $\beta-$ estradiol and bisphenol $\mathrm{A}$ was extracted with $3 \mathrm{~g} \ell^{-1}$ of the polymer, and only $0.3 \mathrm{~g} \ell^{-1}$ was needed to extract more than $90 \%$ of the triclosan. The extraction capacity of the polymer precipitate for the three chemicals was around $10^{-4}$ mol per $1 \mathrm{~g}$ of the precipitate.
\end{abstract}

Key words: environmentally-benign extraction medium, thermoresponsive polymer, poly(vinylmethyl) ether, hazardous chemicals

\section{INTRODUCTION}

Separation and concentration procedures are often important for several stages of laboratory or industrial activities such as analysis of chemicals or recovery of restricted chemicals from wastewaters. Liquid-liquid extraction utilizing an organic solvent such as chloroform or hexane is one of the most suitable techniques for the purpose. However, their use should be eliminated in the interest of environmental friendliness and safety ${ }^{11}$, because such organic solvents themselves are generally toxic or flammable or both. Several aquatic biphasic systems without organic solvents have been studied as safer alternatives ${ }^{2,3}$. Matsubara and her coworkers $^{4-11)}$ reported the successful collection of many hydrophobic materials with precipitate of thermoresponsive polymers. Such polymers have a lower critical solution temperature (LCST); they are watersoluble at temperatures lower than their LCST, but they form gum-like precipitates at higher temperatures ${ }^{12}$. Many hydrophobic solutes are captured in the precipitates, which can easily be separated from the supernatant and redissolved in a very small amount of a solvent such as methanol.

In the present study, we investigated the extraction/concentration efficiencies of some hazardous organic materials with thermoresponsive poly(vinylmethyl) ether (PVME) to evaluate whether the polymer can be applicable for extraction of such chemicals from environmental samples. The PVME is an inexpensive commer- 
cially available material; hence, we selected this polymer. We used three hazardous chemicals, $\beta$-estradiol (3, 17,3-dihydroxy-1,3,5[10]-estratriene; ES), bisphenol A (2,2-bis(4-hydroxyphenyl)propane; BA) and triclosan (2,4,4'-trichloro-2'-hydroxydiphenyl ether; TC) as model solutes. The ES and BA are considered to be endocrine disruptors ${ }^{13)}$ and TC has been reported to be a precursor of dioxin ${ }^{1.4)}$.

\section{MATERIALS AND METHODS}

All the reagents were analytical grade and used without purification. The PVME was purchased from Tokyo Kasei Chemicals as a $30 \%$ aqueous solution. The ES and BA were purchased from Wako Pure Chemicals, and TC was purchased from Japan Ciba Geigy. Sodium $n$-dodecylbenzenesulfonate $\left(\mathrm{C}_{12} \mathrm{LAS}\right)$ was purchased from Tokyo Kasei Chemicals, Japan, polyethyleneglycol tert-octylphenyl ether (Triton X-100; TX100) from Fluka, Switzerland, and humic acid (HMA) from Wako Pure Chemicals, Japan.

The solutions were prepared in a thermostated room at $298 \mathrm{~K}$. A certain volume of aqueous solution containing $1 \times 10^{-7} \sim 1 \times 10^{-4} \mathrm{~mol} \ell^{-1}$ of one of the target chemicals and PVME was placed in a capped glass tube. The initial PVME concentration was kept in the range from 0.3 to $6 \mathrm{~g}^{-1}$. The $\mathrm{pH}$ of the solution was controlled by the addition of sulfuric acid or sodium hydroxide solution. The solution was heated at about 323 $\mathrm{K}$ for $5 \mathrm{~min}$, and then the tube was shaken vigorously for $10 \mathrm{~s}$ by hand. The PVME formed a whitish gumlike precipitate that adsorbed on the walls of the glass tube. After the precipitate and the supernatant were separated by decantation, the precipitate was dissolved in a certain volume of methanol. The concentration of the chemicals in the supernatants and the methanol solutions, which dissolved the PVME precipitate, were measured utilizing a HPLC or GC/MS. The HPLC determination was made using a Shimadzu LC-4A HPLC with a Shimadzu Shim-pack CLC-ODS $(6.0 \mathrm{~mm} \phi \quad \times$ $15 \mathrm{~cm}$ ) reversed-phase column. A mixture of acetonitrile and aqueous $0.02 \mathrm{~mol} \ell^{-1}$ sodium perchlorate solutions $(50 \% / 50 \%$ for ES and BA, and $67 \% / 33 \%$ for TC) was used as the mobile phase. The chemicals were monitored at $240 \mathrm{~nm}$ (TC), $278 \mathrm{~nm}$ (BA) and 280 $\mathrm{nm}$ (ES) with a Shimadzu SPD-2A UV detector. The GC-MS measurements were carried out using an $\mathrm{Ag}$ ilent $6890 \mathrm{~N}$ GC with an Agilent HP-5MS column (0.25 $\mathrm{mm} \phi \times 30 \mathrm{~m})$. The chemicals were monitored with an
Agilent 5973N MS detector; the mass numbers monitored were 272 and 213 for ES, 213 and 228 for BA, and 288 and 290 for TC. The measurement of TC in the experiments for fundamental consideration of PVME extraction and of these three chemicals in the liquid-liquid extraction experiments were carried out with HPLC, while the measurements of BA and ES in the experiments for fundamental consideration of PVME extraction and of these three chemicals in the real environmental samples were carried out with GC/MS.

\section{RESULTS AND DISCUSSION}

The concentrations of the precipitated PVME and the solutes extracted with the PVME precipitate, $[\mathrm{PVME}]_{\text {prec }}$ and [Solute $]_{\text {extr }}$ respectively, are expressed on the basis of the initial volume of the solution as the difference between the concentrations in the aqueous solution before and after heating (expressed with subscripts "init" and "dis", respectively);

$$
\begin{aligned}
& {[\text { PVME }]_{\text {prec }}=[\text { PVME }]_{\text {init }}-[\text { PVME }]_{\text {dis }}} \\
& {[\text { Solute }]_{\text {extr }}=[\text { Solute }]_{\text {init }}-[\text { Solute }]_{\text {dis }}}
\end{aligned}
$$

The reagent blank of the commercially supplied PVME was checked; the blank concentrations for these three chemicals in the product were very small $\left(<<1 \mu \mathrm{g}_{\mathrm{PPME}^{-1}}\right)$ and were negligible.

\section{Experimental Conditions for PVME Precipitation and Extraction}

The extraction behavior of the target chemicals with the PVME precipitate depended on both the efficiency of precipitate formation and the extractability of the chemicals into the precipitate. In order to determine the conditions for the most efficient extraction, we investigated the experimental conditions for precipitate formation and for extractability of the target chemicals.

The aqueous PVME solution has an optical absorbance in the UV region; the recovery of PVME from an acidic aqueous solution as its precipitate was measured using the changes in absorbance at $276 \mathrm{~nm}$ between the supernatant solution after separation of the PVME precipitate and the solution before heating (Figure 1). The recovery of PVME precipitate was not complete and was varied with the change of PVME concentrations, $42 \sim 62 \%$ of the added PVME was pre- 
cipitated from the aqueous solution containing 0.5 $6 \mathrm{~g} \ell^{-1}$ of PVME. The [PVME $]_{\text {prec}}$, which we calculated from the difference in the absorbance of the solutions before and after heating using Eq. 1, agreed well with the amount obtained experimentally by redissolving the precipitate into an equal volume of a fresh aqueous solution. No change in the values was observed when the heating temperature was increased to $343 \mathrm{~K}$. Hence, the change in the amount of precipitate formed was due not to the changes in the experimentally controlled precipitation conditions but an existence of a water-soluble part in the commercially supplied PVME.

The efficiency in the precipitation of PVME should be affected by the conditions of the sample solution; hence, the effects of $\mathrm{pH}$ in solution condition were investigated. The amount of precipitated PVME formed from the aqueous $3 \mathrm{~g} \ell^{-1}$ solution (Recovery ${ }_{\mathrm{PVME}}$ ) was measured as a function of $\mathrm{pH}$ (Figure 2(a)). The amount of PVME precipitate decreased as the solution $\mathrm{pH}$ increased, and no precipitate was formed above $\mathrm{pH}$ 4.5. The recovery of $\mathrm{BA}$ and $\mathrm{TC}$ with $3 \mathrm{~g} \ell^{-1}$ PVME (Recovery $y_{\text {Solute }}$ ) was also determined under several $\mathrm{pH}$ conditions (Figure 2(b)), and it also decreased with increasing $\mathrm{pH}$ and fell to zero when the $\mathrm{pH}$ was higher than 4 (TC) or 4.5 (BA) according to the amount of the precipitate formed. In order to increase the $\mathrm{pH}$ region, at which it was possible to form PVME precipitate and extract the target solutes into it, we measured the salting-out effect of sodium chloride and found that the addition of $0.4 \mathrm{~mol} \ell^{-1}$ sodium chloride had a great effect on the recovery of solutes with PVME in acidic to weakly alkaline solutions. When the solution contained sodium chloride, the $\mathrm{pH}$ region for precipitation widened. The recovery of BA and TC was

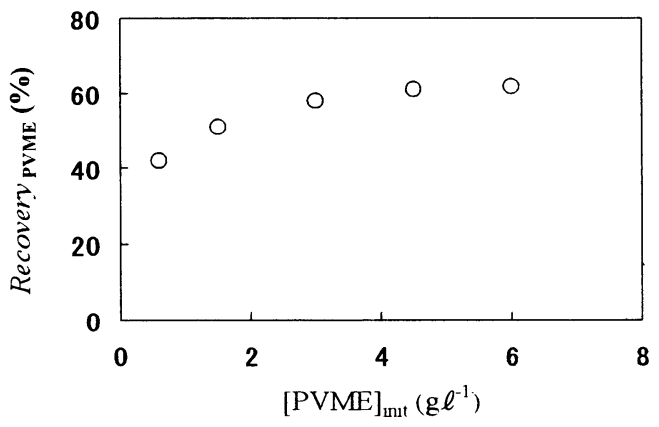

Fig. 1 Recovery of PVME precipitate as a function of $[\mathrm{PVME}]_{\text {init }}$ also improved by the addition of sodium chloride; BA was extracted quantitatively up to $\mathrm{pH} 9$ and $\mathrm{TC}$ to $\mathrm{pH}$ 7. We found that the recovery of the chemicals in the low $\mathrm{pH}$ region was also increased by the addition of the salt. The differences in the curvatures in Fig. 2(b) for different chemicals should be explained by the differences in the distribution equilibria of the chemicals combined with the extractability of the neutral chemicals themselves and/or dissociation/association of the chemicals with hydrogen ion at the appropriate $\mathrm{pH}$ conditions.

To utilize this extraction medium to the recovery of target materials in real environmental water samples, it is important to consider the effects of coexist-
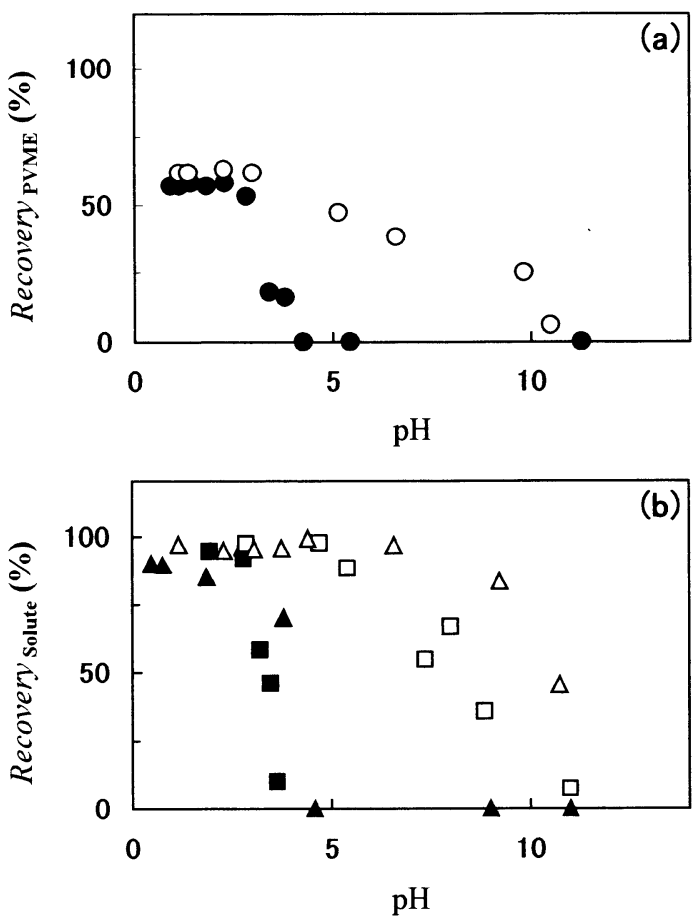

Fig. 2 Changes in the recovery of (a) PVME as a precipitate from $3 \mathrm{~g} \ell^{-1}$ solution, and (b) $\mathrm{BA}$ (triangle) and $\mathrm{TC}$ (square) with $3 \mathrm{~g}^{-1}$ PVME, as a function of $\mathrm{pH}$. Open symbols correspond to the data obtained in the presence of $0.4 \mathrm{~mol} \ell^{-1}$ sodium chloride, and closed symbols correspond to the data obtained in the absence of any additional salt. The $\mathrm{pH}$ was measured at $298 \mathrm{~K}$ before the solution was heated 
ing materials in the samples on the efficiencies in the formation of precipitate and in the extractability of target chemicals. The recovery of the PVME precipitate (Recovery $_{\mathrm{PVME}}$ ) and of $\mathrm{TC}$ in the precipitate (Recovery ${ }_{\mathrm{TC}}$ ) was examined as a function of coexisting $\mathrm{C}_{12} \mathrm{LAS}$, TX100 and HMA concentration, which are typical coexisting organic materials in polluted water systems. The recovery of the precipitate (Figures 3(a)-3(c)) was decreased when the concentration of the coexisting materials became higher, especially in the case of $\mathrm{C}_{12} \mathrm{LAS}$. To increase the efficiency in formation of precipitate, the salting-out effect of sodium chloride was measured. As seen in Figures 3(a) and 3(c), the recovery of the precipitate was improved by addition of $0.4 \mathrm{~mol} \ell^{-1}$ sodium chloride up to mostly similar to that obtained without the coexisting materials, although the improvement was still not high enough when the PVME concentration was $0.6 \mathrm{~g} \ell^{-1}$. The recovery of TC with extraction by the precipitate formed from 0.6 and $3 \mathrm{~g} \ell^{-1}$ PVME was also measured (Figures 3(d)-3(f)). The extractability of $\mathrm{TC}$ decreased drastically to zero in the case of $0.6 \mathrm{~g} \ell^{-1}$ PVME extraction system in the presence of $\mathrm{C}_{12}$ LAS. It was also found that no quantitative extraction for TC was established with $0.6 \mathrm{~g} \ell^{-1}$ PVME in the presence of HMA. These decreases in the recovery of TC were observed when the recovery of the precipitate decreased as similar tendency. The changes in the recovery of TC with PVME by addition of $0.4 \mathrm{~mol} \ell^{-1}$ sodium chloride were investigated; the results are shown in Figures 3(d) and 3(f). The extractability of TC was improved by the salting-out effect, more than $80 \%$ of TC was extracted by PVME precipitate from the aqueous solution containing these organic coexisting materials.

Several studies aimed at determining the effects of additives such as salt or acid on thermoresponsive polymer extraction have been carried out; however, most of the studies were designed to determine changes in $\operatorname{LCST}^{7,10,1215)}$, and consideration of the effects of dissolved additives on the changes in aggregation efficiency at the different $\mathrm{pH}$ regions was limited. The addition of sulfuric acid up to $0.3 \sim 0.6 \mathrm{~mol}^{-1}$ prior to heating has been reported for the extraction of an ionassociate complex of malachite green cation and molybdophosphate anion with PVME or poly $(N$-isopropylacrylamide $)^{5}$. The $\mathrm{pH}$ range for successful extraction in these previous studies is somewhat different than that observed in the present study.

\section{Extractability of Three Organic Chemicals into PVME Precipitates}

Figure 4 shows the relationship between the concentrations of pollutants extracted and the concentration remaining in the supernatant solution when the initial PVME concentration was $3 \mathrm{~g} \ell^{-1}$. The relationship between the amounts of the chemicals extracted into the PVME precipitate and the concentrations remaining in the supernatant was found to be proportional in the lower concentration region, although the curves deviated from linearity in the higher concentra-
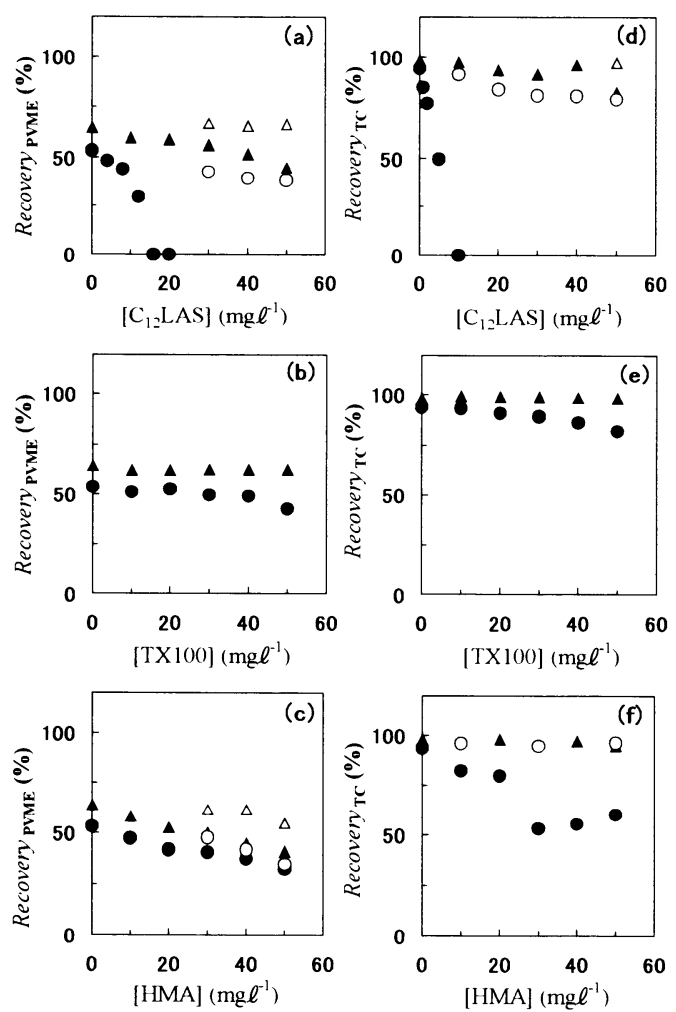

Fig. 3 Changes in the recovery of PVME as a precipitate (left side) and of TC with PVME precipitate (right side) as a function of coexisting $\left[\mathrm{C}_{12} \mathrm{LAS}\right]((\mathrm{a})$ and (d)), [TX100] ((b) and (e)) and [HMA] ((c) and (f)). The PVME concentrations are $0.6 \mathrm{~g}^{-1}$ (circles) and $3 \mathrm{~g} \ell^{-1}$ (triangles), and the open symbols correspond to the data obtained in the presence of 0.4 mol $\ell^{-1}$ sodium chloride, and closed symbols correspond to the data obtained in the absence of any additional salt 
tion region of the chemicals. The shapes of the curvatures can be explained by Langmuir's adsorption equation;

$[\text { Solute }]_{\text {extr }}=\alpha_{\max } \beta[\mathrm{PVME}]_{\text {prec }}[\text { Solute }]_{\text {dis }}\left(1+\beta[\text { Solute }]_{\text {dis }}\right)$

Here, $\alpha_{\max }$ is the saturated amount for adsorption onto

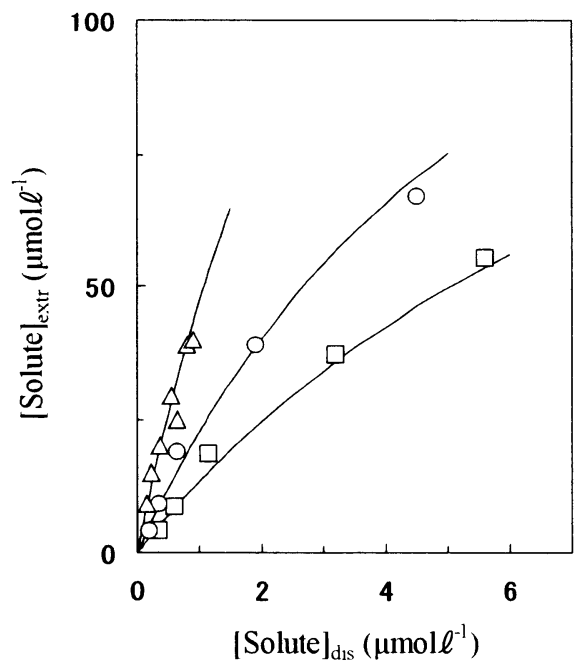

Fig. 4 Adsorption curves of BA (squares), ES (circles) and $\mathrm{TC}$ (triangles) with $3{\mathrm{~g} \ell^{-1}}^{-1}$ PVME precipitate as a function of the solute concentration in the supernatant. The lines were calculated by plugging the values of $\alpha_{\max }$ and $\beta$ in Table 1 into Eq. 3
$1 \mathrm{~g}$ of precipitated PVME, and $\beta$ is the adsorption coefficient for the solute. The value of $[\mathrm{PVME}]_{\text {prec }}$ under the present experimental condition was obtained from Fig. 1. The calculated values of $\alpha_{\max }$ and $\beta$ are listed in Table 1. The extractabilities of the chemicals with PVME increased in the order $\mathrm{BA}<\mathrm{ES}<\mathrm{TC}$; and the maximum capacity of the PVME precipitate for these chemicals was about $10^{-4} \mathrm{~mol}$ per $1 \mathrm{~g}$ of PVME precipitate. As can be seen in Figure 4, the concentration factor of the solutes is highest in the very low [Solute $]_{\text {dis }}$ region. We calculated the minimum concentration of PVME precipitate that could extract more than 90 $\%$ of the solutes by introducing the values of $\alpha_{\max }$ and $\beta$ in Table 1 into Eq. 4.

$$
[\text { Solute }]_{\mathrm{extr}} /[\text { Solute }]_{\mathrm{dis}}=\alpha_{\max } \beta[\mathrm{PVME}]_{\mathrm{prec}}=9
$$

The expected maximum concentration factors of the solutes $\left(R_{90 \% \text { (calcd) }}\right)$ were calculated, the values obtained are summarized in Table 1.

We also obtained the solute concentration factors directly by measuring the solute recovery at different PVME concentrations. The initial concentrations of PVME ranged from 0.3 to $6 \mathrm{~g} \ell^{-1}$, and the initial solute concentrations were lower than $5 \times 10^{-5} \mathrm{~mol} \ell^{-1}$. Figure 5 shows the results of the measurements. The extractability of TC was very high; the recovery was greater than $95 \%$ in the concentration range examined. In contrast, the recovery of $\mathrm{ES}$ and $\mathrm{BA}$ decreased when the PVME concentration was less than $1.5 \mathrm{~g} \ell^{-1}$. The precipitate formed with $0.3 \mathrm{~g}$ of PVME could be dissolved in only $1 \mathrm{~m} \ell$ of methanol; hence,

Table 1 Adsorption coefficients and concentration factors for three organic toxicants in the PVME precipitate system

\begin{tabular}{|c|c|c|c|c|}
\hline Solutes & $\begin{array}{c}\alpha_{\max } \\
\left(\operatorname{mol} g_{\text {PVME }} \text { prec }^{-1}\right)\end{array}$ & $\begin{array}{c}\beta \\
\left(1 \mathrm{~mol}^{-1}\right)\end{array}$ & 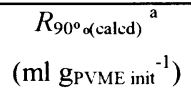 & $\begin{array}{c}R_{\left.90^{\circ} \text { obbsd }\right)}{ }^{b} \\
\left(\mathrm{ml} \mathrm{g}_{\mathrm{PVME} \text { init }}{ }^{-1}\right)\end{array}$ \\
\hline$\overline{B A}$ & $8.5 \times 10^{-5}$ & $9.7 \times 10^{4}$ & $5.0 \times 10^{2}$ & $6.7 \times 10^{2}$ \\
\hline ES & $9.9 \times 10^{-5}$ & $1.5 \times 10^{5}$ & $7.9 \times 10^{2}$ & $6.7 \times 10^{2}$ \\
\hline $\mathrm{TC}$ & $1.4 \times 10^{-4}$ & $2.3 \times 10^{5}$ & $1.3 \times 10^{3}$ & $>3.3 \times 10^{3}$ \\
\hline
\end{tabular}

a. Calculated maximal concentration factor (the ratio between the initial volume of water and the initial weight of PVME) to achieve quantitative ( $>90 \%$ ) extraction of the chemicals.

b. Experimentally observed (Fig. 5) maximal concentration factor (the ratio between the initial volume of water and the initial weight of PVME) to achieve quantitative ( $>90 \%)$ extraction of the chemicals. 
the concentration factor was theoretically at least hundreds times, even without any procedure, such as evaporation, for enrichment of the solvent.

\section{Comparison of the PVME System and Conven- tional Liquid-Liquid Systems}

The extractabilities of the three chemicals with hexane and dichloromethane, which are the organic solvents typically utilized for liquid-liquid extraction of organic pollutants, were measured and compared with the concentration efficiencies obtained with the PVME system. The distribution constant $\left(K_{\mathrm{d}}=[\text { Solute }]_{\text {org }} /[\text { Solute }]_{\text {aq }}\right)$

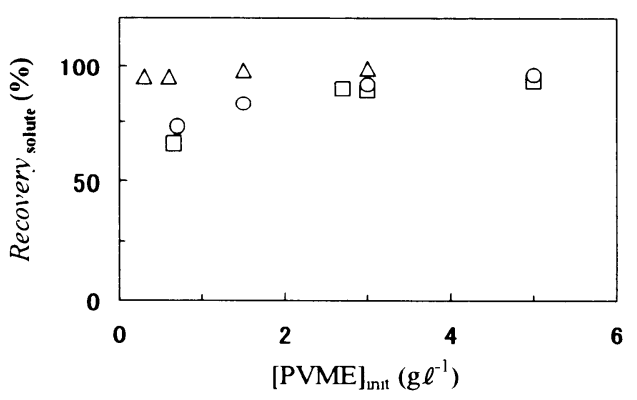

Fig. 5 Changes in the recovery of BA (squares), ES (circles) and TC (triangles) into PVME precipitate as a function of initial PVME concentration. The $\mathrm{pH}$ of the solutions was maintained at 2 , and no additional salt was added to the solutions obtained for the three chemicals in the two organic solvent systems are listed in Table 2. Using the values of $K_{\mathrm{d}}$, we calculated possible concentration factors for quantitative extraction $(>90 \%)$ of the chemicals in these systems. Because the partition constant was low, concentration by extraction of BA and ES with hexane was impossible, and the concentrations obtained with dichloromethane were not high enough (3.4 for BA and 39 for ES). The concentration factor of 300 and 1000 is theoretically possible for TC with hexane and dichloromethane; however, the phase separation in these systems becomes difficult because the volume of the organic solvent becomes very small by the mutual solubility. The concentration factor obtained with the PVME extraction system was about 500 (for BA and ES) and 1000 (for TC), as shown in Table 1. These values of concentration factor in the PVME system were higher than those in the solvent extraction system especially for BA and ES, although the units of the factors are different in the two systems.

It is also noteworthy that the extractability differences between these three solutes are larger in the liquid-liquid systems (more than $10^{5}$-fold in hexane-water and more than $3 \times 10^{2}$-fold in dichloromethane-water from $\mathrm{BA}$ to $\mathrm{TC}$ ) than in the PVME system (about 3-5-fold from BA to TC). The very small differences between the extractabilities of solutes in the PVME system indicate that this system is suitable for separation of groups of solutes having similar characteristics, for example, a recovery of several kinds of restricted chemicals from wastewater.

Table 2 Distribution constants and concentration factors for three organic toxicants in two conventional liquidliquid systems

\begin{tabular}{ccccc}
\hline & \multicolumn{2}{c}{ Hexane-water $^{\mathrm{a}}$} & \multicolumn{2}{c}{ Dichloromethane-water $^{\mathrm{a}}$} \\
Solutes & $K_{\mathrm{d}}$ & $R_{90^{\circ}{ }^{\mathrm{b}}}$ & $K_{\mathrm{d}}$ & $R_{90^{\circ}{ }^{\mathrm{b}}}$ \\
& $(-)$ & $(-)$ & $(-)$ & $(-)$ \\
\hline BA & $4.1 \times 10^{-2 \mathrm{c}}$ & $4.5 \times 10^{-3}$ & $3.0 \times 10^{1}$ & 3.4 \\
ES & $6.2 \times 10^{-1 \mathrm{c}}$ & $6.9 \times 10^{-2}$ & $3.5 \times 10^{2}$ & $3.9 \times 10^{1}$ \\
TC & $2.8 \times 10^{3}$ & $3.1 \times 10^{2}$ & $9.1 \times 10^{3}$ & $1.0 \times 10^{3}$ \\
\hline
\end{tabular}

a. The $\mathrm{pH}$ of the aqueous phase was maintained $\mathrm{pH} 2$ with sulfuric acid.

b. Calculated maximal concentration factor (the ratio between the volume of the water and the volume of the organic solvent) to achieve quantitative (>90\%) extraction of the chemicals

c. In hexane - aqueous $5 \%$ methanol solution. 


\section{Extraction of BA, ES and TC in River Water Sam- ples}

In order to learn the applicability of the present pretreatment technique for real environmental water, the analysis of BA, ES and TC in some river waters were demonstrated. The samples were collected from three rivers in Kanto plain area. Surface waters were collected using a polyethylene beaker and stocked in capped $3 \ell$ glass bottles. The samples were brought back to the laboratory, and then filtered using a glass microfibre filter with $0.45-\mu \mathrm{m}$ pore (Whatman, GF-C) within 3 hours after collection. The filtered solutions were stored in a refrigerator. A $200 \mathrm{~m} \ell$ of the samples was taken into a $500 \mathrm{~m} \ell$ capped glass flask, acidified to $\mathrm{pH} 2$ by addition of sulflic acid, and added PVME up to $0.3 \mathrm{~g} \ell^{-1}$. The solution was heated $333 \mathrm{~K}$ for $10 \mathrm{~min}$, then, the flask was shaken vigorously for $30 \mathrm{~s}$ to form precipitate. The precipitate was separated from the supernatant by decantation; the precipitate was dissolved with $2 \mathrm{~m} \ell$ of methanol after the supernatant was taken away from the flask. The chemicals in the methanol solution were analyzed by a GC-MS without any concentration and/or clean up treatment. The results obtained were summarized in Table 3 . The blank for these three chemicals, which were contaminated during the extraction and concentration procedures, was also measured; the values were about a few $n g \ell^{-1}$ for all three chemicals. The values in Table 3 were corrected one by subtraction of the blank. Although the concentrations obtained are somewhat erroneous, the levels of these three chemicals are in the ranges of the concentrations reported ${ }^{16-18)}$.

\section{CONCLUSION}

The PVME precipitate that formed when an aqueous polymer-containing solution was heated to temperatures higher than $305 \mathrm{~K}$ quantitatively extracted BA, ES and TC from aqueous solutions into the innersphere. The extractability of the chemicals decreased with increasing solution $\mathrm{pH}$; however, quantitative extraction was maintained up to neutral $\mathrm{pH}$ region by the addition of $0.4 \mathrm{~mol} \ell^{-1}$ sodium chloride. The efficiencies in the formation of precipitate and in the extraction of chemicals were decreased by the addition of an anionic surfactant $\mathrm{C}_{12}$ LAS while no such effect was observed by the addition of TX100 or HMA. The inefficiency with $\mathrm{C}_{12}$ LAS was improved by the addition of sodium chloride. One gram of PVME precipitate can capture about $10^{-4} \mathrm{~mol}$ of these chemicals, and only $1.5 \mathrm{~g}$ of the polymer is needed to capture more than $90 \%$ of the chemicals from $1 \ell$ aqueous solution. The high concentration factors indicate that the extraction system may be an useful alternative to liquid-liquid extraction with organic solvents for several purposes of applications in extraction/separation/concentration of trace materials from aquatic samples.

\section{REFERENCES}

1) Bradley, D.R.: Organic reactions enter a new phase. Chem. Ind., 86 (1999)

2) Huddleston, J.G., Willauer, H.D., Griffin, S.T. and Rogers, R.D.: Aqueous polymeric solutions as environmentally benign solvent extraction media. Ind. Eng. Chem. Res., 38, 2523-2539 (1999)

3) Hinze, W.L. and Pramauro, E.: A critical review of surfactant-mediated phase separations (cloud-point

Table 3 The concentration levels of BA, ES and TC in some environmental waters measured utilizing the present pretreatment method

\begin{tabular}{lcccc}
\hline \multicolumn{1}{c}{ Sampling site } & $\mathrm{N}^{\mathrm{a}}$ & $\begin{array}{c}\mathrm{BA} \\
\left(\mathrm{ng} \ell^{-1}\right)\end{array}$ & $\begin{array}{c}\mathrm{ES} \\
\left(\mathrm{ng} \ell^{-1}\right)\end{array}$ & $\begin{array}{c}\mathrm{TC} \\
\left(\mathrm{ng} \ell^{-1}\right)\end{array}$ \\
\hline Ohtsu R. (at Hidori Bridge) & 4 & $95 \pm 26$ & $59 \pm 19$ & $83 \pm 16$ \\
Inari R. (at Mikazuki Bridge) & 2 & $76 \pm 14$ & $34 \pm 11$ & $89 \pm 27$ \\
Kozakura R. (breast of Mt. Tsukuba) & 2 & $\mathrm{nd}^{\mathrm{b}}$ & $\mathrm{nd}^{\mathrm{b}}$ & $\mathrm{nd}^{\mathrm{b}}$ \\
\hline
\end{tabular}

a: Number of samples

b: Not detected. 
extractions): Theory and applications. Crit. Rev. Anal. Chem., 24, 133-177 (1993)

4) Matsubara, C., Izumi, S., Takamura, K., Yoshioka, H. and Mori, Y.: Determination of trace amounts of phosphate in water after preconcentration using a thermally reversible polymer. Analyst, 118, 553556 (1993)

5) Matsubara, C., Kikuchi, N., Denpouya, I. and Takamura, K.: A new preconcentration method of hydrophobic substances in water using polyvinylmethylether as a thermo-responsive polymer. Application to spectrophotometric determination of a trace amount of phosphate in natural water. Chem. Lett., 849850 (1993)

6) Saitou, T., Ohyama, T., Takamura, K., Sakurai, T., Kaise, T. and Matsubara, C.: Extraction of iron(II)tris(1,10-phenanthrolinato) chelate into the polymer phase formed from an aqueous solution of $\operatorname{poly}(\mathrm{N}$ isopropylacrylamide). Anal. Sci., 13, 1-4 (1997)

7) Saitou, T., Ohyama, T., Sakurai, T., Kaise, T., Takamura, K., Suzuki, Y. and Matsubara, C.: Polymermediated extraction of 8-quinolinolato metal chelates for the determination of metal ions in water with graphite furnace atomic absorption spectrometry. Talanta, 46, 541-550 (1998)

8) Saitou, T., Haga, M., Sakurai, T., Kaise, T. and Matsubara, C.: Polymer-mediated extraction for microplate reader spectrophotometric determination of ppb-level copper(II) ion in water. Anal. Sci., 14, 929-933 (1998)

9) Saitou, T., Ohkubo, S. and Matsubara, C.: Polymermediated extraction of porphyrinato chelates for a spectrophotometric approach to sub-ppt level detection of metal ions. Chem. Lett., 151-152 (1999)

10) Saitou, T., Yoshida, Y., Matsudo, T., Fujiwara, S., Dobashi, A., Iwaki, K., Suzuki, Y. and Matsubara, C.: Concentration of hydrophobic organic com- pounds by polymer-mediated extraction. Anal. Chem., 71, 4506-4512 (1999)

11) Saitou, T., Yoshida, Y. and Matsubara, C.: Concentration of nonylphenol and its polyethoxylated derivatives by polymer-mediated extraction. $J$. Chromatogr., 891, 69-74 (2000)

12) Schild, H. G. and Tirrell, D. A.: Microcalorimetric detection of lower critical solution temperatures in aqueous polymer solutions. J. Phys. Chem., 94, 4352-4356 (1990)

13) "Environmental Hormones" Edited by Environment Agency of Japan, pp115-117, Kankyo Shinbunsha, Tokyo (1998)

14) Kanetoshi, A., Ogawa, H., Katsura, E. and Kaneshima, H.: Chlorination of irgasan DP300 and formation of dioxins from its chlorinated derivatives. J. Chromatogr., 389, 139-153 (1987)

15) Horne, R.A., Almeida, J.P., Day, A.F. and Yu, N. T.: Macromolecule hydration and the effect of solutes on the cloud point of aqueous solutions of polyvinyl methyl ether: A possible model for protein denaturation and temperature control in homeothermic animals. J. Colloid Interface Sci., 35, 77-84 (1971)

16) River Bureau, Ministry of Land, Infrastructure and Transport: "Results of the field investigation study on endocrine disruptors in aquatic environments 2002”, Press Release on Oct. 24, 2003

17) Sabaliunas, D., Webb, S.F., Hauk, A., Jacob, M. and Eckhoff, W. S.: Environmental fate of Triclosan in the River Aire basin, UK. Water Res., 37, 31453154 (2003)

18) Wanami, K., Shimazu, T. and Tamura, M.: Study on endocrine disruptors in Tokyo's rivers (10): The variations of estrogen and estrogen-like activity in the Tama river. Annual Rep. Tokyo Metropolitan Res. Inst. Environ. Protection, 66-74 (2002) 